\title{
A self-packaged capacitive humidity sensor with low leakage loss
}

\author{
Huiyang Yu ${ }^{1 \text { a) }}$, Jianqiu Huang ${ }^{2}$, Chaojin $\mathrm{Xing}^{2}$, and Yuzhi Chu ${ }^{1}$ \\ ${ }^{1}$ College of Computer Science and Technology, Nanjing Tech University, \\ No. 30, Puzhu South Road, Nanjing 211800, China \\ ${ }^{2}$ Key Laboratory of MEMS of Ministry of Education, Southeast University, \\ No. 2, Sipailou, Nanjing 210096, China
}

a)yhy_njtech@163.com

Abstract: A capacitive humidity sensor based on a flip-chip self-packaged process is presented. An air gap was applied to separate two electrodes of the sensitive capacitor and reduce the leakage loss especially in high humidity environment. Two typical structures of the sensor were developed. Both static and dynamic properties of fabricated sensors were evaluated. The dielectric loss tangent of both structures were less than $8 \%$ even at $95 \% \mathrm{RH}$. Meanwhile, both response time and recovery time of the sensors were short because one side of the sensitive layer was open to the air.

Keywords: humidity sensor, capacitive sensor, flip-chip, leakage loss Classification: Micro- or nano-electromechanical systems

\section{References}

[1] T. A. Blank, et al.: "Recent trends of ceramic humidity sensors development: A review," Sens. Actuator B-Chem. 228 (2016) 416 (DOI: 10.1016/j.snb.2016. 01.015).

[2] A. Tripathy, et al.: "Role of morphological structure, doping, and coating of different materials in the sensing characteristics of humidity sensors," Sensors 14 (2014) 16343 (DOI: 10.3390/s140916343).

[3] R. Puers: "Capacitive sensors: When and how to use them," Sens. Actuator A-Phys. 37-38 (1993) 93 (DOI: 10.1016/0924-4247(93)80019-D).

[4] S. Westerlund and L. Ekstam: "Capacitor theory," IEEE Trans. Dielectr. Electr. Insul. 1 (1994) 826 (DOI: 10.1109/94.326654).

[5] J. Wang, et al.: "Study on dielectric properties of humidity sensing nanometer materials," Sens. Actuator B-Chem. 108 (2005) 445 (DOI: 10.1016/j.snb.2004. 11.089).

[6] T. Islam, et al:: "Investigation of the electrical characteristics on measurement frequency of a thin-film ceramic humidity sensor," IEEE Trans. Instrum. Meas. 65 (2016) 694 (DOI: 10.1109/TIM.2015.2506302).

[7] T. Zhang, et al:: “Analysis of dc and ac properties of humidity sensor based on polypyrrole materials," Sens. Actuator B-Chem. 131 (2008) 687 (DOI: 10. 1016/j.snb.2007.12.059).

[8] P. Vooka and G. Boby: "An improved capacitance-to-digital converter for leaky capacitive sensors,” IEEE Sensors J. 15 (2015) 6238 (DOI: 10.1109/JSEN. 2015.2454531).

[9] P. Vooka and B. George: “Capacitance-to-digital converter for leaky capacitive 
sensors," Electron. Lett. 52 (2016) 456 (DOI: 10.1049/el.2015.3962).

[10] F. Reverter and C. Òscar: "A microcontroller-based interface circuit for lossy capacitive sensors,” Meas. Sci. Technol. 21 (2010) 065203 (DOI: 10.1088/ 0957-0233/21/6/065203).

[11] G. G. Zhang, et al.: "Critical temperatures in thermocompression gold stud bonding," J. Appl. Phys. 102 (2007) 063519 (DOI: 10.1063/1.2783974).

[12] L. F. Chen, et al.: Microwave Electronics: Measurement and Materials Characterization (John Wiley \& Sons, 2004) 1st ed. 8.

[13] M. Matsuguchi, et al.: "Stability and reliability of capacitive-type relative humidity sensors using crosslinked polyimide films," Sens. Actuator B-Chem. 52 (1998) 53 (DOI: 10.1016/S0925-4005(98)00255-X).

[14] U. Kang and K. D. Wise: "A high-speed capacitive humidity sensor with on-chip thermal reset," IEEE Trans. Electron Devices 47 (2000) 702 (DOI: 10. 1109/16.830983).

[15] Y. Luo, et al:: "Research of radiosonde humidity sensor with temperature compensation function and experimental verification," Sens. Actuator A-Phys. 218 (2014) 49 (DOI: 10.1016/j.sna.2014.07.015).

[16] J.-H. Kim, et al.: "High-performance capacitive humidity sensor with novel electrode and polyimide layer based on MEMS technology," Microsyst. Technol. 16 (2010) 2017 (DOI: 10.1007/s00542-010-1139-0).

[17] C.-L. Zhao, et al:: "A fully packaged CMOS interdigital capacitive humidity sensor with polysilicon heaters," IEEE Sensors J. 11 (2011) 2986 (DOI: 10. 1109/JSEN.2011.2154325).

[18] J.-H. Kim, et al:: "Capacitive humidity sensors based on a newly designed interdigitated electrode structure," Microsyst. Technol. 18 (2012) 31 (DOI: 10. 1007/s00542-011-1373-0).

[19] A. Rivadeneyra, et al.: "Design and characterization of a low thermal drift capacitive humidity sensor by inkjet-printing," Sens. Actuator B-Chem. 195 (2014) 123 (DOI: 10.1016/j.snb.2013.12.117).

[20] Z. Chen and C. Lu: "Humidity sensors: A review of materials and mechanisms," Sens. Lett. 3 (2005) 274 (DOI: 10.1166/sl.2005.045).

[21] R. Fenner and E. Zdankiewicz: "Micromachined water vapor sensors: A review of sensing technologies," IEEE Sensors J. 1 (2001) 309 (DOI: 10.1109/7361. 983470).

\section{Introduction}

Capacitive sensors draw lasting attraction of researches due to the advantages of low power consumption, high performances and long-stability [1, 2, 3]. An ideal capacitive device is a reactive element. However, the resistance of lead wires and the leakage loss in dielectric materials increase the total power consumption and degrade the properties of capacitors [4].

In capacitive humidity sensors, water tends to condense inside porous sensitive materials under the influence of Kelvin effect so that leakage current rises significantly with the humidity [5]. The leakage current deteriorates both nonlinearity and stability of humidity sensors and makes the capacitance varies with frequency [6, 7]. Many efforts have been made to develop circuits for leaky capacitive sensors $[8,9,10]$, but few researches on low-leakage structures were conducted. 
In this paper, an air gap was inserted into a sandwich capacitor to avoid the leakage loss in a capacitive humidity sensor. A flip-chip bonding process was applied to fabricate the sensor. The sensor was self-packaged and showed low leakage even at high humidity.

\section{Structure and fabrication}

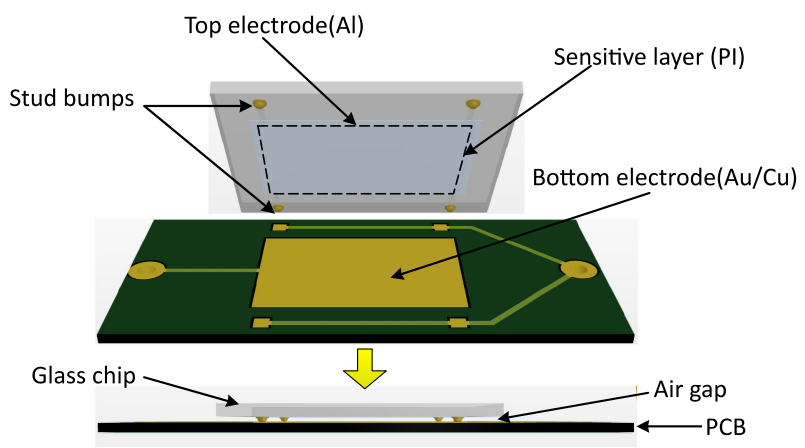

Fig. 1. Schematic of the humidity sensor.

Fig. 1 shows the structure of the capacitive humidity sensor. The sensitive capacitor consists of a top electrode, a sensitive layer, an air gap and a bottom electrode. The top electrode and sensitive layer locate on a glass chip. The bottom electrode locates on a PCB board. The glass chip and the PCB board are connected by stud bumps.

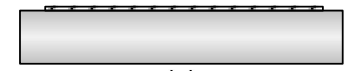

(a)

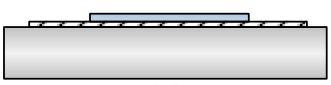

(b)

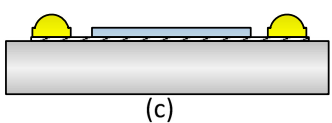

$\square$ Glass

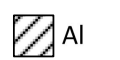

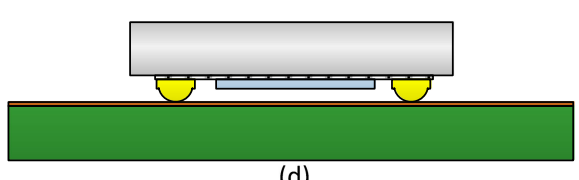

(d)

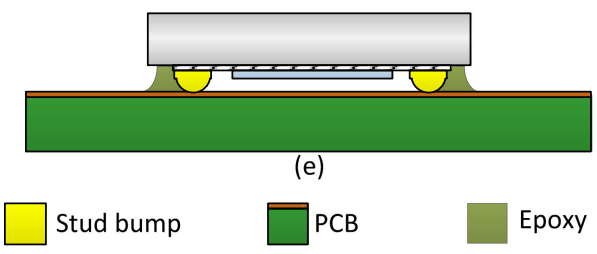

Fig. 2. Fabrication process of the humidity sensor.

The structure was realized by a micro-fabrication process and a flip-chip package process as shown in Fig. 2. First, a $0.5 \mu \mathrm{m}$ thick aluminum layer was sputtered on a glass substrate and patterned as the top electrode. Next, a polyimide film (ZKPI-600-II, POME Sci-tech Inc.) with the thickness of $1 \mu \mathrm{m}$ was spun on the top electrode and patterned as a sensitive layer. Because ZKPI-600-II polyimide is photosensitive, it was exposed with i-line lithography $\left(150 \mathrm{~mJ} / \mathrm{cm}^{2}\right)$ and cured with a four-step heating process $\left(180^{\circ} \mathrm{C}\right.$ for 1 hour, $250^{\circ} \mathrm{C}$ for 1 hour, $300^{\circ} \mathrm{C}$ for 1 hour and $350^{\circ} \mathrm{C}$ for 0.5 hour). After that, gold stud bumps were fabricated on the pads of the top electrode with a typical wire bonding process [11]. Then the glass chip was flip-chip bonded onto a PCB board where the bottom electrode was fabricated. The temperature and pressure used to assemble were $270^{\circ} \mathrm{C}$ and $15 \mathrm{Mpa}$ respectively. 
Finally the sensor was epoxy-underfilled at each corner of the chip to strength the structure.

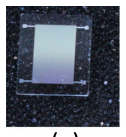

(a)

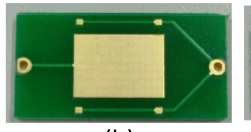

(b)

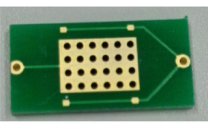

(c)

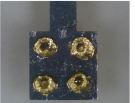

(d)

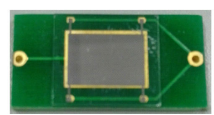

(e)

Fig. 3. Photograph of the humidity sensor. (a) glass chip, (b) PCB board without vent holds, (c) PCB board with vent holds, (d) pad with gold stud bumps, (e) bonded sensor.

The fabricated glass chip and PCB boards used to assemble are shown in Fig. 3(a)-(c). Different PCB boards (with or without vent holes) were used for comparison. Fig. 3(d) exhibits the gold stud bumps on the pad of the top electrode. To improve the stability of contact, a $2 \times 2$ array of bumps were applied on each pad. The photograph of a bonded sensor is shown in Fig. 3(e). The sizes of the sensitive capacitor are $5.5 \mathrm{~mm} \times 8 \mathrm{~mm}$.

\section{Results}

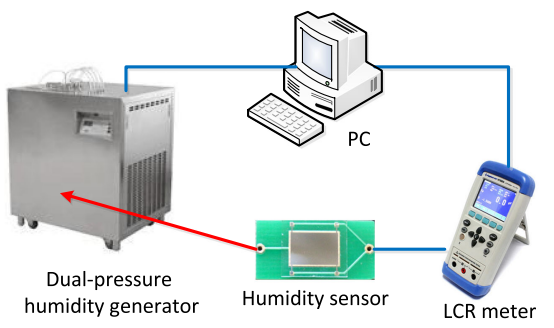

(a)

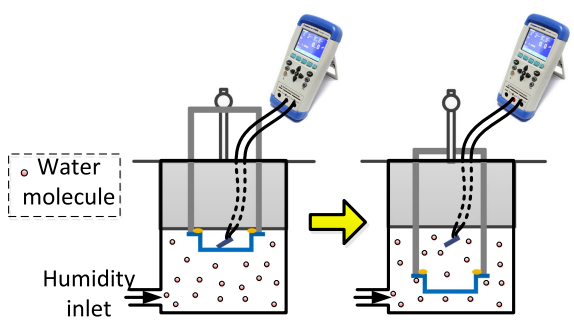

(b)

Fig. 4. Test systems of the sensor. (a) static test system, (b) dynamic test system.

Both static and dynamic properties of the sensors were tested in this paper. Static tests were performed used the equipment described in Fig. 4(a). A dual-pressure humidity generator controlled by a computer was used to simulate the humidity environment. A LCR meter was used to test the sensitive capacitance and the leakage loss of the sensor. The results were collected by a computer. The test frequency of the LCR meter was $100 \mathrm{kHz}$. As to dynamic tests, a mechanical setup was developed to make a step change in humidity. The mechanical setup was 
located in the test chamber of the dual-pressure humidity generator. As shown in Fig. 4(b), at the beginning, the sensor was sealed in a cap and the humidity outside the cap was changed. Then the cap was quickly ejected downloads to the bottom of the chamber and the sensor was exposed to a different humidity environment immediately. At the same time, responses of the humidity sensor were measured by a LCR meter and recorded to evaluate the dynamic properties of the sensor.

Static test results of proposed humidity sensors are shown in Fig. 5(a). Structures with different PCB boards (with or without vent holds) are used for comparison. Here, we call the structure without vent holes as Type 1 and that with vent holes as Type 2 . The performances of both structures are described in Table I.

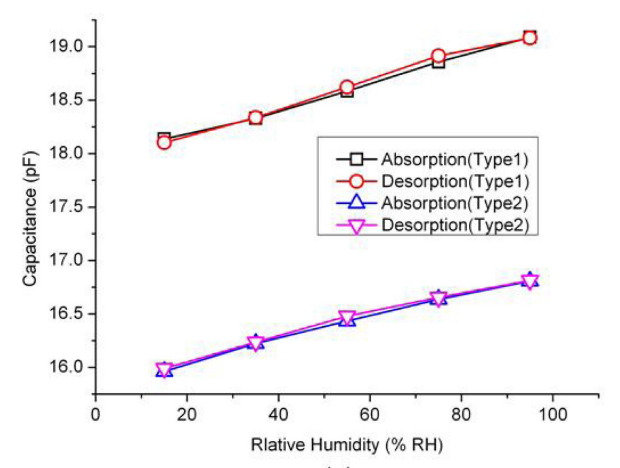

(a)

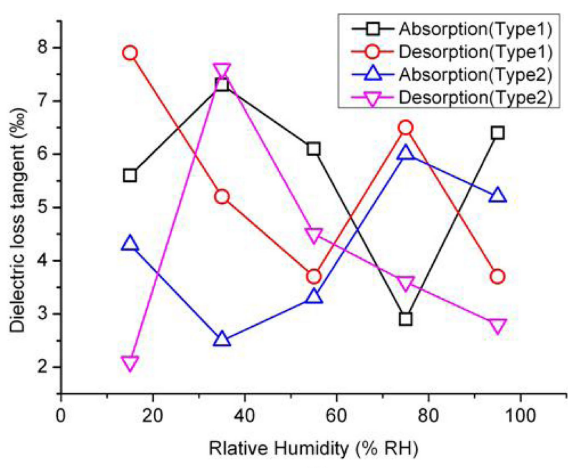

(b)

Fig. 5. Static responses of the humidity sensors. (a) capacitive responses, (b) dielectric loss responses.

It is noted that the sensitive capacitance of Type 2 is less than that of Type 1 because the effective area of capacitor is reduced by vent holes. As a result the sensitivity of Type 2 is smaller than that of Type 1. However the hysteresis of Type 2 is better than that of Type 1 with the aid of vent holes.

Table I. Static properties of the sensors

\begin{tabular}{cccc}
\hline & Sensitivity & Hysteresis & Linearity \\
\hline Type 1 & $12 \mathrm{fF} / \% \mathrm{RH}$ & $\pm 2.4 \% \mathrm{RH}$ & $3.3 \%$ \\
Type 2 & $11 \mathrm{fF} / \% \mathrm{RH}$ & $\pm 2.2 \% \mathrm{RH}$ & $3.9 \%$ \\
\hline
\end{tabular}

As a plate capacitor, the sensitive capacitance $\mathrm{C}$ of the sensor is calculated as:

$$
C=\frac{\varepsilon_{a i r} \varepsilon A}{\varepsilon_{a i r} t+\varepsilon g}
$$

where $\varepsilon_{\text {air }}$ and $\varepsilon$ are the permittivity of air and the sensitive material respectively. $A$ is the area of the sensitive capacitor, $t$ is the thickness of the sensitive material and $g$ is the gap between the sensitive material and the bottom electrode.

As variation of $\varepsilon_{a i r}$ with humidity is almost negligible, the relative change of the sensitive capacitance can be expressed as:

$$
\frac{\Delta C}{C}=\frac{1}{1+\frac{g \varepsilon}{t \varepsilon_{\text {air }}}} \times \frac{\Delta \varepsilon}{\varepsilon}
$$


It is obvious that the relative change of the sensitive capacitance increases with the thickness $t$ and decreases with the gap $g$. A narrow gap can lead to large capacitance and high sensitivity. If $g=0$, the sensor will become a standard sandwich structure and the relative change of sensitive capacitance will be independent of geometrical parameters. In this paper, stud bumps were utilized and the gap g was about $20 \mu \mathrm{m}$. To get a narrower gap, electroplated bumps can be applied in the further work.

The leakage loss of a capacitor can be characterized by the dielectric loss tangent. As the capacitor is not an ideal element, a complex permittivity $\varepsilon=\varepsilon^{\prime}+\mathrm{j} \varepsilon^{\prime \prime}$ should be used to describe the device. The dielectric loss tangent is used to describe the energy dissipation of the dielectric material [12]. It is defined as:

$$
\tan \delta_{e}=\frac{\varepsilon^{\prime \prime}}{\varepsilon^{\prime}}
$$

The relationship between the dielectric loss tangent and the humidity is showed in Fig. 5(b). In both structures the dielectric loss tangent is not affected by the humidity. Even at $95 \% \mathrm{RH}$ the dielectric loss tangent is less than $8 \%$. It means that the leakage loss is negligible and the humidity sensor can be treated as an ideal capacitor.

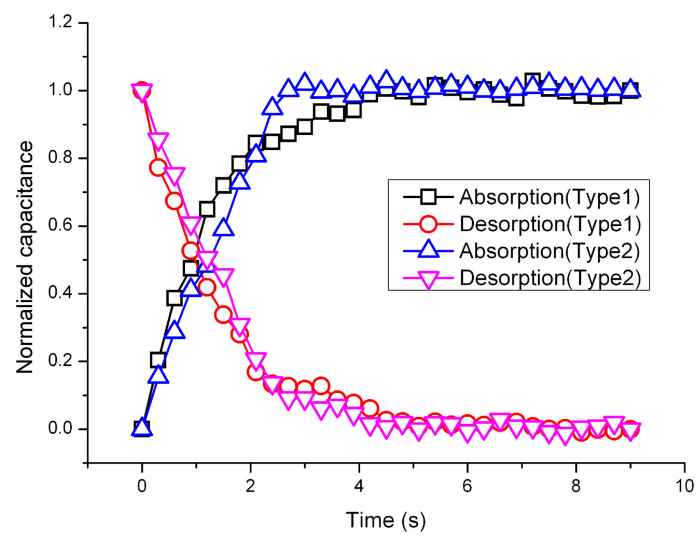

Fig. 6. Dynamic responses of the humidity sensors.

Dynamic responses of the humidity sensors are shown in Fig. 6. Corresponding response time and recovery time for a $90 \%$ step are summarized in Table II. The recovery process is slower than the response process in each structure. With the aids of vent holes, vapor can be exchanged more quickly so that Type 1 exhibit faster dynamic responses. As one side of the sensitive layer is open to the air, the responses of both structures are rapid.

Table II. Dynamic properties of the sensors

\begin{tabular}{ccc}
\hline & Response time (s) & Recovery time (s) \\
\hline Type 1 & 3.0 & 3.5 \\
Type 2 & 2.1 & 2.7 \\
\hline
\end{tabular}


Table III. Comparison of different reported polyimide capacitive sensors

\begin{tabular}{lllllll}
\hline Literature & Structure & $\begin{array}{l}\text { PI } \\
\text { Thick } \\
(\boldsymbol{\mu m})\end{array}$ & $\begin{array}{l}\text { Sensitivity } \\
(\mathbf{f F} / \mathbf{\%} \text { RH) }\end{array}$ & $\begin{array}{l}\text { Response } \\
\text { time (s) }\end{array}$ & $\begin{array}{l}\text { Recovery } \\
\text { time (s) }\end{array}$ & Packaged \\
\hline Matsuguch [13] & Sandwich & - & $130 \sim 170$ & - & - & no \\
Kang [14] & Sandwich & 2 & 30.0 & $\begin{array}{l}\text { 1(air } \\
\text { channel) }\end{array}$ & - & no \\
Yi [15] & Sandwich & 1 & 428 & $1.3($ heating) & - & no \\
Kim [16] & Sandwich & 0.1 & 506 & 6 & - & no \\
Zhao [17] & IDE & 2.4 & 1.36 & $32($ heating) & $24($ heating) yes \\
Kim [18] & Wavy IDE & 4 & 10.8 & - & - & no \\
Rivadeneyra [19] & IDE & 75 & 4.5 & $>1000$ & $>1000$ & no \\
This work & Sandwich & 1 & $11 \sim 12$ & $2.1 \sim 3$ & $2.7 \sim 3.5$ & yes \\
\hline
\end{tabular}

Traditionally, there are two kinds of capacitive structures: Interdigited (IDE) structures and sandwich (parallel plate) structures [1, 20, 21]. IDE electrodes are widely used in gas sensors because of the simple structure and easy fabrication process. However, IDE structures usually exhibit small sensitive capacitance and low sensitivity. To increase the sensitive of IDE structures, a thick sensitive layer should be used $[17,18]$. In exchange, the dynamic properties of the sensors will be degraded. In contrast, sandwich structures usually show large sensitive capacitance and high sensitivity. A thin sensitive layer improves both sensitivity and dynamic properties of sandwich structures but dielectric leakage problems should be considered. Furthermore, a porous top electrode should be fabricated to allow gases to pass though it [20]. With special materials or techniques to realize the porous layer, the fabricated processes of sandwich structures are usually complicated.

Table III shows properties of some typical capacitive humidity sensors with polyimide as the sensitive material. It shows that the sensitivity of sandwich capacitive humidity sensors ranges from $\sim 10 \mathrm{fF} / \% \mathrm{RH}$ to $\sim 100 \mathrm{fF} / \% \mathrm{RH}$, while the sensitivity of IDE capacitive humidity sensors ranges from $\sim 1 \mathrm{fF} / \% \mathrm{RH}$ to $\sim 10 \mathrm{fF} / \% \mathrm{RH}$. In traditional sandwich structures, a breathable top electrode is critical for the properties of capacitive humidity sensors and the sensitivity tends to decrease with the thickness of the sensitive layer. To accelerate the diffusion of gas molecules, air channels inside the sensitive layer [14] or heating resistors under the capacitor [15] were developed. In IDE structures the sensitivity tends to increase with the thickness of the sensitive layer. To increase the sensitivity of IDE capacitive humidity sensors, a wavy structure and a thick polyimide $(4 \mu \mathrm{m})$ were used by Kim et al. [18]. But the response time of the humidity sensor should also increase with the thickness of the sensitive layer. We must trade off static properties against dynamic properties in IDE structures.

Compared with traditional capacitive humidity sensors, the presented structure shows lower sensitivity than typical sandwich structures but higher sensitivity than IDE structures. To improve the sensitivity of the presented structure, electroplated 
bumps can be applied to realize a narrow gap. The relationship between the sensitive capacitance and the air gap can be evaluated by Eq. (1). As shown in Fig. 7 , if the air gap decreases to $2 \mu \mathrm{m}$, the sensitive capacitance will increase to about $300 \mathrm{pF}$. The sensitivity will increase as well. Moreover, with the aid of the air gap, dynamic properties of the presented structure are improved. Both air gap and air channels can improve the dynamic properties, but the fabrication process of air channels are more complicated [14]. Furthermore, it is noted that most of the reported capacitive humidity sensors are not packaged. Normally, the package will significantly degrade the properties of humidity sensors [17]. The effect of package should be taken into account when the presented self-paged structure is compared with other unpackaged structures. At last, the dielectric leakage in capacitive humidity sensors was neglected by most of the researchers. However, as the humidity rise, the leakage conduction of the sensitive material increases [4]. Because of the leakage current, capacitive humidity sensors tend to degrade or even fail in high humidity environment. In this work, ultra low dielectric loss was realized by the presented structure at $95 \% \mathrm{RH}$. We believe it can work well even at higher humidity.

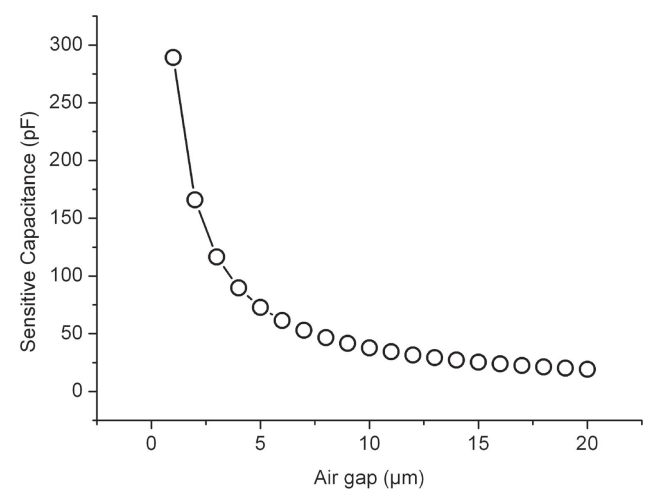

Fig. 7. Calculated sensitive capacitance with the air gap.

\section{Conclusion}

A capacitive humidity sensor with low leakage loss is proposed. The sensor is selfpackaged and two typical structures of the sensor are reported. Test results show that the sensitivity, hysteresis and linearity of both structures are $12 \mathrm{fF} / \%$ vs. 11 fF/\%RH, $\pm 2.4 \%$ RH vs. $\pm 2.2 \%$ RH, and $3.3 \%$ vs. $3.9 \%$ respectively. Both structures exhibit low dielectric loss and fast response properties.

\section{Acknowledgments}

This work was partially supported by the Natural Science Foundation of Jiangsu Province (BK20161009), the National Natural Science Foundation of China (61771136), and the open research fund of Key Lab of Broadband Wireless Communication and Sensors Network Technology of (Nanjing University of Posts and Telecommunications), Ministry of Education (NYKL201505). 